\title{
Development in the drug delivery tools that transport medically active biomolecules
}

\begin{abstract}
The drug-delivery tool that transport medically active molecules to diseased cells, in a precise manner, have grew much consideration in past decades. Supramolecular selfassembled systems plays an important role in nanotechnology, biotechnology, and regenerative medicine. ${ }^{1}$ Based on self-assembly approach various drug-delivery systems have been developed for example polymers, ${ }^{2}$ micelles, ${ }^{3}$ vesicles, ${ }^{4}$ nanoparticles ${ }^{5}$ and vesicular-supported particles. ${ }^{6}$ Among these developed systems, silica particles based delivery tool have become popular as biocompatible alternatives. ${ }^{7}$ Most sophisticated mesoporous silica nanoparticle (MSNs) have widely used ${ }^{8}$ due to their applicability to release drug molecule in controlled in particular cells using internal stimuli, such as $\mathrm{pH}^{9}$ and enzymes ${ }^{10}$ or external stimuli such as light, ${ }^{11}$ redox properties ${ }^{12}$ and temperature..$^{13}$ The most discovered approaches to controlled drug-release were based on MSN carriers through surface functionalisation, with biomolecule responsive gates, $\mathrm{pH}$ - or photo-triggered release from hollow MSN. ${ }^{14}$ However, these methods are challenging, and suffer from limitations due to the low tissue-penetration-depth of light. ${ }^{15}$
\end{abstract}

\author{
Volume I Issue 3 - 2017
}

\author{
Sheshanath V Bhosale,' Mahmood \\ Aljabri,' Aman Amanpreet Hundal,' Sam \\ Leslie Jackson,' 'Duong Duc La,' Mina \\ Salimimarand,' Sidhanath V. Bhosale, ${ }^{2}$ \\ Sheshanath $\vee$ Bhosale' \\ 'RMIT University, Australia \\ ${ }^{2}$ CSIR Indian Institute of Chemical Technology, India
}

Correspondence: Sheshanath $\vee$ Bhosale, School of Science, RMIT University, Australia,

Email sheshanath.bhosale@rmit.edu.au

Received: July 19, 2017 | Published: September 13, 2017

\section{Introduction}

Thus, there is a need to develop ideal transport system that should be designed to control the release of loaded drug to the target areas, ${ }^{16}$ thereby increasing its local concentration, bioavailability and prolonging its retention. To overcome these issues, recently, silica nanoparticles have generated a significant amount of interest because of their intrinsic properties as shown to be biocompatible alternatives. ${ }^{17}$ Based on their characteristic few sophisticated delivery systems have been developed and employed using mesoporous silica nanoparticles. ${ }^{18}$ However, these delivery systems have their own advantages, but they suffer from their own limitations, such as poor chemical or thermal elimination ${ }^{19}$ and also difficulties in controlling targeting and the well-organised release of the drug, as well as rapid elimination by the immune system. ${ }^{20}$

Keeping this in mind recently we prepared well-like cavity-so called 'yoctowells $\left(1 \mathrm{yL}=8 \mathrm{~nm}^{3}\right.$ that is, $\left.10^{-24} \mathrm{~L}\right)$,' by two step selfassembly, firstly covalently bound porphyrin to the silica surface and bolaaphiphiles around the base porphyrins. ${ }^{21}$ For selection of porphyrin as a base component has two important advantageous, first is it can use as a handle while drug loading and drug release and second is penetration of particles in particular cells can be monitored easily with fluorescence of base porphyrin. Yoctowells are easy to prepare and can be tuned based on application one wishes. ${ }^{22-27}$ We have used these yoctowells for various applications such as The most fascinating property of yoctowells is their ability to induce the formation of wellfilling "nanocrystals" in dilute aqueous solutions, for example cyclic edge amphiphiles ${ }^{24}$ or neurotransmitters. ${ }^{25}$ Due to yoctowells unique rigid and hydrophobic nature have gifted outstanding abilities to selectively bind various guests entities.

Taking advantageous of yoctowell capability to encapsulate drug molecule, recently, we have demonstrate the use these hydrophobic wells as simple model systems for the encapsulation FDA approved anti-cancer Doxorubcin (DOX) biologically active molecules and their release was monitored by biological stimuli i.e. $\mathrm{pH} .{ }^{27}$ Typically, two step-self assembled yoctowells were firstly functionalised with ammonium group produced positive rim at the top of the wells, upon addition of DOX 2 molecule, the positive rim used for capping of the yoctowells by addition of an anionic-porphyrin 3 by electrostatic interaction. After confirmation by UV-vis absorption and fluorescence spectroscopy, we then studied controlled release of the DOX and capping porphyrin $\mathbf{3}$ from the yoctowells by $\mathrm{pH}$ control. This system provide the first report of effectiveness of the sustain release of the DOX 2 molecule selectively from the yoctowells, offers prospective for development of a new generation of drug-delivery system for practical application. However, this developed yoctowell drugdelivery system relied on non-active silica nanoparticles, and has limitations for targeted drug delivery. To overcome this literature support our hypothesis to transport systems based on magnetic nanoparticles which can be ideal for the controlled release of loaded drug to target areas, thereby increasing its local concentration and bioavailability, and prolonging its retention. As human tissues are transparent to magnetic fields, ${ }^{28}$ thus use of magnetic field can be a good substitute for exact and targeted release of drugs. ${ }^{29}$

Knowing the capabilities of yoctowells, and the importance of targeted delivery of cancer drugs, herein, we transport system on magnetic silica nanoparticles and their application for encapsulation and $\mathrm{pH}$-controlled release of drug molecules is verified (Figure 1). ${ }^{30-35}$ To demonstrate the process of the responsive mechanised yoctowells, mitoxantrone (MTZ) was chosen for the release experiments. MTZ is from a member of the anthracycline antibiotic family, and is a powerful anticancer drug against different malignancies for example malignant tumours, several forms of leukemia, as well as ovarian and breast cancer. ${ }^{26} \mathrm{MTZ}$ has major clinical value due to its apparent lower risk of cardiotoxic effects, as compared with DOX 2.36-39 


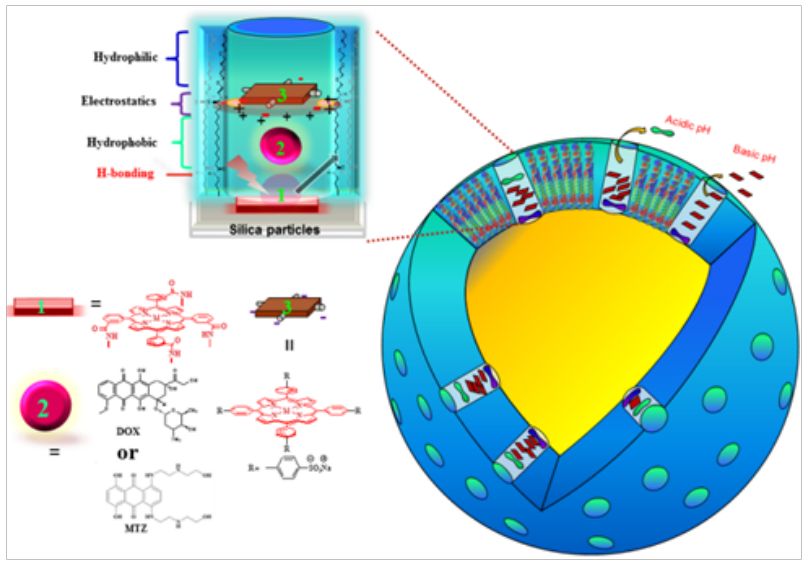

Figure I Graphical illustration to demonstrate encapsulation of drug molecules 2 and capping porphyrin on the top to cover the wells and their step-by-step release by manipulation naturally occurring stimulus.

\section{Conclusion}

In summary, we demonstrate two novel approaches, first is preparation yoctowells on the magnetic silica nanoparticles for targeted delivery and reusable applications, and second is the encapsulation drug DOX or MTZ and their release by manipulating naturally occurring stimuli in vivo, that is, $\mathrm{pH}$. Thus, we believe in future the usefulness of the sustained release of the DOX or MTZ molecule from yoctowells may provide potential tool for the development of a new generation of targeted drug-delivery systems. Thus, designer yoctowells, may act as tiny chemical reactors or alternatives in vivo drug-delivery systems by manipulating the interactions between drug molecules and the walls of yoctowell gaps and/or base porphyrin, and are thought to provide a useful supramolecular tool and could open new opportunities in the realm for targeted therapies.

\section{Acknowledgements}

S.V.B. (RMIT) acknowledges the Australian Research Council for funding through the Future Fellowship Scheme (FT110100152).

\section{Conflict of interest}

The author declares no conflict of interest.

\section{References}

1. JM Lehn. Toward self organization and complex matter. Science. 2000;295(5564):2400-2403.

2. Ke C, Destecroix H, Crump MP, et al. A simple and accessible synthetic lectin for glucose recognition and sensing. Nat Chem. 2012;4(9):718-723.

3. Aida T, Meijer EW, Stupp SI. Functional supramolecular polymers. Science. 2012;335(6070):813-817.

4. Zhang X, Rehm S, Safont Sempere MM, et al. Vesicular perylene dye nanocapsules as supramolecular fluorescent $\mathrm{pH}$ sensor systems. Nat Chem. 2009;1(2):623-629.

5. Mirkin CA, Letsinger RL, Mucic RC, et al. A DNA-based method for rationally assembling nanoparticles into macroscopic materials. Nature. 1996;382(6592):607-609.

6. Bhosale S, Sisson AL, Talukdar P, et al. Photoproduction of proton gradients with pi-stacked fluorophore scaffolds in lipid bilayers. Science. 2006;313(5783):84-86.
7. Allen TM, Cullis PR. Drug delivery systems: entering the mainstream. Science. 2004;303(5665):1818-1822.

8. Uhrich KE, Cannizzaro SM, Langer RS, et al. Polymeric systems for controlled drug release. Chem Rev. 1999;99(11):3181-3198.

9. Lee CC, MacKay JA, Fréchet JM, et al. Designing dendrimers for biological applications. Nat Biotechnol. 2005;23(12):1517-1526.

10. Okuda T, Tominaga K, Kidoaki S. Time programmed dual release formulation by multilayered drug loaded nanofiber meshes. J Controlled Release. 2010;143(2):258-264.

11. Kataoka K, Harada A, Nagasaki Y. Block copolymer micelles for drug delivery: design, characterization and biological significance. Adv Drug Delivery Rev. 2001;47(1):113-131.

12. Moon JJ, Suh H, Bershteyn A, et al. Interbilayer crosslinked multilamellar vesicles as synthetic vaccines for potent humoral and cellular immune responses. Nat Mater. 2011;10(3):243-251.

13. Brigger I, Dubernet C, Couvreur P. Nanoparticles in cancer therapy and diagnosis. Adv Drug Delivery Rev. 2002;54(5):631651.

14. Ashley CE, Carnes EC, Phillips GK, et al. The targeted delivery of multicomponent cargos to cancer cells by nanoporous particle supported lipid bilayers. Nat Mater. 2011;10(5):389-397.

15. Kortesuo P, Ahola M, Karlsson S, et al. Silica xerogel as an implantable carrier for controlled drug delivery - evaluation of drug distribution and tissue effects after implantation. J Biomaterials. 2000;21(2):193198.

16. Li Z, Barnes JC, Bosoy A, et al. Mesoporous silica nanoparticles in biomedical applications. Chem Soc Rev. 2012;41(7):2590-2605.

17. Rim HP, Min KH, Lee HJ, et al. pH Tunable calcium phosphate covered mesoporous silica nanocontainers for intracellular controlled release of guest drugs. Angew Chem Int Ed. 2011;50(38):8853-8857.

18. Potat A, Ross BP, Liu J, et al. Enzyme-Responsive Controlled Release of Covalently Bound Prodrug from Functional Mesoporous Silica Nanospheres. Angew Chem Int Ed. 2012;51(50):12486-1289.

19. Angelos S, Yang YW, Khashab NM, et al. Dual-controlled nanoparticles exhibiting AND logic. J Am Chem Soc. 2009;131(32):11344-11346.

20. Lai CY, Trewyn BG, Jeftinija DM, et al. A mesoporous silica nanosphere based carrier system with chemically removable CdS nanoparticle caps for stimuli-responsive controlled release of neurotransmitters and drug molecules. J Am Chem Soc. 2003;125(15):4451-4459.

21. Sauer AM, Schlossbauer A, Ruthardt N, et al. Role of Endosomal Escape for Disulfide-Based Drug Delivery from Colloidal Mesoporous Silica Evaluated by Live-Cell Imaging. Nano Lett. 2010;10(9):3684-3691.

22. Schlossbauer A, Warncke S, Gramlich PME, et al. A Programmable DNA-Based Molecular Valve for Colloidal Mesoporous Silica. Angew Chem Int Ed. 2010;49(28):4734-4737.

23. Wang C, Li Z, Cao D, et al. Cover Picture: One Hundred Years of Vitamins-A Success Story of the Natural Sciences. Angew Chem Int Ed. 2012;51(52):5460-5465

24. Lammertyn J, Peirs A, De Baerdemaeker J, et al. Light penetration properties of NIR radiation in fruit with respect to non-destructive quality assessment. Biol Technol. 2000;18(2):121-132.

25. Peer D, Karp JM, Hong S, et al. Nanocarriers as an emerging platform for cancer therapy. Nat Nanotechnol. 2007;2(12):751-760.

26. Radin $\mathrm{S}$. Tissue rections to controlled release silica xerogel carriers. In: LeGeros, RZ LeGeros, editors. Proceeding of the 11th International symposium on Ceramics in Medicine. New York, USA: World Scientific Publishing; 1998. p. 529-532. 
27. Li Z, Barnes JC, Bosoy A, et al. Mesoporous silica nanoparticles in biomedical applications. Chem Soc Rev. 2012;41(7):2590-2605.

28. Nagesh Kolishettia, Shanta Dhar, Pedro M Valencia, et al. Engineering of self-assembled nanoparticle platform for precisely controlled combination drug therapy. Proc Natl Acad Sci USA. 2010;107(42):17939-17944.

29. Okuda T, Tominaga K, Kidoaki S. Time-programmed dual release formulation by multilayered drug-loaded nanofiber meshes. J Controlled Release. 2010;143(2):258-264.

30. Bhosale SV, Langford SJ. Recent developments in utilising yoctowells for investigations in nanospace. Chem Soc Rev. 2012;41(5):1637-1651.

31. Li G, Bhosale SV, Wang T, et al. Nanowells on silica particles in water containing long-distance porphyrin heterodimers. J Am Chem Soc. 2003;125(35):10693-10702.

32. Bhosale S, Bhosale S, Wang T, et al. Slow Motion, Trapping, and Sorting of Water- and Chloroform-Soluble Porphyrins in Nanowells. J Am Chem Soc. 2004;126(40):13111-13118.

33. Andrew C Benniston, Anthony Harriman, Peiyi Li, et al. TemperatureInduced Switching of the Mechanism for Intramolecular Energy Transfer in a $2,2^{`}: 6^{`}, 2^{\prime}$ "-Terpyridine-Based Ru(II)-Os(II) Trinuclear Array. Chem Commun. 2005;127(8):2559-2564.
34. Bhosale SV, Jani CH, Langford SJ. Chemistry of naphthalene diimides. Chem. 2008;37(2):331-343.

35. Bhosale S, Bhosale S, Wang $\mathrm{T}$, et al. Hydrophobic and Hydrophilic Yoctowells as Receptors in Water. $J$ Am Chem Soc. 2006;128(8):2156-2157.

36. Bhosale SV, Bhosale SV. Yoctowells as a simple model system for the encapsulation and controlled release of bioactive molecules. Sci Rep. 2013;3:1982.

37. Tiihonen J, Hari R, Kajola M, et al. Magnetoencephalographic $10-\mathrm{Hz}$ rhythm from the human auditory cortex. Neurosci Lett. 1991;129(2):303-305.

38. Lu F, Popa A, Zhou S, et al. Iron oxide-loaded hollow mesoporous silica nanocapsules for controlled drug release and hyperthermia. Chem Commun. 2013;49(97):11436-11438.

39. Bhosale SV. Yoctowell Cavities on Magnetic Silica Nanoparticles for $\mathrm{pH}$ Stimuli-Responsive Controlled Release of Drug Molecules. Chem Eur J. 2014;20(18):5253-5257. 